\title{
Pebble Delivery for Inside-Out Planet Formation
}

\author{
Xiao $\mathrm{Hu}^{1}$ Jonathan C. Tan ${ }^{1}$ and Sourav Chatterjee ${ }^{2}$ \\ ${ }^{1}$ Department of Astronomy, University of Florida, 32611, Gainesville, Florida, USA. \\ email: ustcxhu@ufl.edu, jt@astro.ufl.edu \\ ${ }^{2}$ Center for Interdisciplinary Exploration and Research in Astrophysics (CIERA) \\ Department of Physics \& Astronomy, Northwestern University, Evanston, IL 60208, USA. \\ email: sourav. chatterjee@northwestern.edu
}

\begin{abstract}
Inside-Out Planet Formation (IOPF; Chatterjee \& Tan 2014, hereafter CT14) is a scenario for sequential in situ planet formation at the pressure traps of retreating dead zone inner boundaries (DZIBs) motivated to explain the many systems with tightly packed inner planets (STIPs) discovered by Kepler. The scenario involves build-up of a pebble-dominated protoplanetary ring, supplied by radial drift of pebbles from the outer disk. It may also involve further build-up of planetary masses to gap-opening scales via continued pebble accretion. Here we study radial drift \& growth of pebbles delivered to the DZIB in fiducial IOPF disk models.
\end{abstract}

Keywords. planetary systems: protoplanetary disks, planetary systems: formation

\section{Introduction}

Kepler observations show STIPs are very common. The compact, well-aligned, but largely non-resonant architectures of these systems challenge formation scenarios involving migration of already-formed planets from the outer disk (e.g., Hands et al. 2014).

Chiang \& Laughlin (2013) discussed aspects of in situ formation. Hansen \& Murray $(2012$; 2013) studied STIP formation from a disk of protoplanets, requiring initial conditions with highly enriched solid surface densities above the minimum mass solar nebula.

CT14 proposed the IOPF scenario to link enrichment of solids in the inner disk by pebble drift with simultaneous and sequential formation of planets at the pressure maximum associated with the transition from a dead zone to a magneto-rotational instability (MRI)-dominated zone in the very inner disk, perhaps first set by the thermal ionization of alkali metals at $\sim 1200 \mathrm{~K}$. Here we calculate the radial drift timescale of different sizes of pebbles starting from various outer disk locations. We then couple this to a simple growth model for the pebbles. These are the first steps towards calculation of the global pebble supply rate to the DZIB, which will control the rate of IOPF.

\section{Drag laws, radial drift velocity and pebble growth}

Following Armitage (2010), we define the gas drag frictional timescale of a pebble of mass $m_{p}$ moving at speed $v_{p}$ relative to gas as $t_{\text {fric }}=\left(m_{p} v_{p}\right) / F_{D}$. We consider four drag regimes (first is Epstein; others are Stokes regimes depending on Reynolds no., Re):

$$
t_{\text {fric }}= \begin{cases}\rho_{p} a_{p} /\left(\rho_{g} v_{p}\right) & \text { if } a_{p}<9 \lambda / 4 \\ 2 \rho_{p} a_{p}^{2} /(9 \nu \rho) & \text { if } a_{p}>9 \lambda / 4 \text { and } \operatorname{Re}<1 \\ \left(\rho_{p} a_{p} /\left[9 \rho_{g} v_{p}\right]\right)\left(2 a v_{p} / \nu\right)^{0.6} & \text { if } 1<\operatorname{Re}<800 \\ 8 \rho_{p} a_{p} /\left(1.32 \rho_{g} v_{p}\right) & \text { if } \operatorname{Re}>800,\end{cases}
$$


where $a_{p} \equiv a_{p, 1} \mathrm{~cm}$ is pebble radius, $\rho_{p} \equiv \rho_{p, 3} 3 \mathrm{~g} \mathrm{~cm}^{-3}$ is pebble density \& $\rho_{g}$ is gas density. $\nu \approx \lambda c_{s}$ is molecular viscosity, where $\lambda$ is mean free path of gas molecules \& $c_{s}$ is sound speed. In terms of normalized frictional time, $\tau_{\text {fric }} \equiv \Omega_{K} t_{\text {fric }}$, where $\Omega_{K}=$ $\left(G m_{*} / r^{3}\right)^{1 / 2}$ is orbital angular frequency at radius $r \equiv r_{\mathrm{AU}} \mathrm{AU}$ about star of mass $m_{*} \equiv m_{*, 1} M_{\odot}$, radial drift speed is

$$
v_{r, p} \simeq-k_{P}\left(c_{s} / v_{K}\right)^{2}\left(\tau_{\text {fric }}+\tau_{\text {fric }}^{-1}\right)^{-1} v_{K},
$$

where $k_{P}$ describes the disk's pressure profile via $P=P_{0}\left(r / r_{0}\right)^{-k_{P}}$ (with fiducial value of 2.55) and $v_{K}$ is the Keplerian velocity. We integrate $v_{r, p}$ to obtain the radial location of pebbles as a function of time $r_{p}(t)$. In the Epstein regime and the Stokes regime with $\operatorname{Re}<1, v_{r, p}$ is solved for analytically. When $\operatorname{Re}>1$, we solve numerically.

We also consider a simple pebble growth model. The pebble accumulates all smallgrain dust material along its path within its physical cross section while in the Epstein regime. In the Stokes regimes we assume such accumulation is completely inefficient by deflection of the grains due to the pressure gradients of diverted gas streamlines.

\section{Results and Discussion}

Figure 1a shows examples of radial drift in a fiducial disk model with accretion rate $\dot{m} \equiv \dot{m}_{-9} 10^{-9} M_{\odot} \mathrm{yr}^{-1}$ and Shakura-Sunyaev viscosity parameter $\alpha \equiv \alpha_{-3} 10^{-3}$ (assumed constant outwards from the DZIB). Pebbles with radius $0.1 \mathrm{~cm}$ take about $10^{5} \mathrm{yr}$ to reach the inner region if starting from $10 \mathrm{AU}$. This timescale reduces to about $10^{4} \mathrm{yr}$ and $10^{3} \mathrm{yr}$ for $1 \mathrm{~cm}$ and $10 \mathrm{~cm}$ sized pebbles, respectively.

Figure $1 \mathrm{~b}$ shows that varying the accretion rate over a range of a factor of 100 around the fiducial value has only modest effects on the drift timescales. Changing the value of $\alpha$ over a similar range has a somewhat larger effect. For most disk radii shown here we are in the Epstein regime with $\tau_{\text {fric }} \ll 1$. In this case, $v_{r, p}$ is

$$
v_{r, p} \simeq 1.92 a_{p, 1} \rho_{p, 3} \gamma_{1.4}^{8 / 5} \kappa_{10}^{2 / 5} \alpha_{-3}^{3 / 5} m_{*, 1}^{-2 / 5}\left(f_{r} \dot{m}_{-9}\right)^{-1 / 5} r_{\mathrm{AU}}^{1 / 5} \mathrm{~m} \mathrm{~s}^{-1},
$$

where $\gamma \equiv 1.4 \gamma_{1.4}$ is power-law exponent of the barotropic equation of state, $\kappa \equiv$ $\kappa_{10} 10 \mathrm{~cm}^{2} \mathrm{~g}^{-1}$ is disk opacity, and $f_{r} \equiv 1-\sqrt{r_{*} / r}$ (where $r_{*}$ is stellar radius). So pebbles starting at $10 \mathrm{AU}$ drift in faster in lower accretion rate disks and in more viscous disks. At $\sim 100$ AU this simple dependence on $\dot{m}$ breaks down as $\tau_{\text {fric }} \gtrsim 1$.

Figure 1c shows the results for the pebble growth model, starting from $0.1 \mathrm{~cm}$ sizes. Such pebbles grow quickly, shortening delivery times from $100 \mathrm{AU}$ to a few $\times 10^{4} \mathrm{yr}$.

Following CT14, the mass in solids (initially dust) inside disk radius $r_{1}$ is

$$
M_{s}\left(<r_{1}\right) \approx 0.178 f_{s,-2} \gamma_{1.4}^{-4 / 5} \kappa_{10}^{-1 / 5} \alpha_{-3}^{-4 / 5} m_{*, 1}^{1 / 5} \dot{m}_{-9}^{3 / 5} r_{1, \mathrm{AU}}^{7 / 5} M_{\oplus},
$$

(correcting minor typo in eq. 30 of CT14) where $f_{s} \equiv f_{s,-2} 0.01$ is the ratio of solid to gas mass. The gap-opening planetary mass at $r_{0}$ is

$$
M_{G} \approx 5.67 \phi_{G, 0.3} \gamma_{1.4}^{4 / 5} \kappa_{10}^{-1 / 5} \alpha_{-3}^{4 / 5} m_{*, 1}^{3 / 10}\left(f_{r} \dot{m}_{-9}\right)^{2 / 5} r_{0, \mathrm{AU}}^{1 / 10} M_{\oplus},
$$

based on fraction $\phi_{G} \equiv \phi_{G, 0.3} 0.3$ of viscous-thermal mass (Zhu et al. 2013). If a fraction, $\epsilon_{p} \equiv \epsilon_{p, 0.5} 0.5$, of disk solid mass forms pebbles that are accreted by the innermost forming planet with only minor gas accretion (as expected at $T \simeq 1200 \mathrm{~K} \mathrm{DZIB}$ conditions), then the required feeding radius of this first gap-opening mass planet is

$$
r_{1} \approx 19.4 \phi_{G, 0.3}^{5 / 7} \gamma_{1.4}^{8 / 7} \kappa_{10}^{2 / 7}\left(f_{s,-2} \epsilon_{p, 0.5}\right)^{-5 / 7} \alpha_{-3}^{8 / 7} m_{*, 1}^{1 / 14} \dot{m}_{-9}^{-1 / 7} r_{0, \mathrm{AU}}^{1 / 14} \mathrm{AU} .
$$

With fiducial disk parameters and $r_{0}=0.1 \mathrm{AU}$, we have $r_{1}=16.5 \mathrm{AU}$. The drift time 


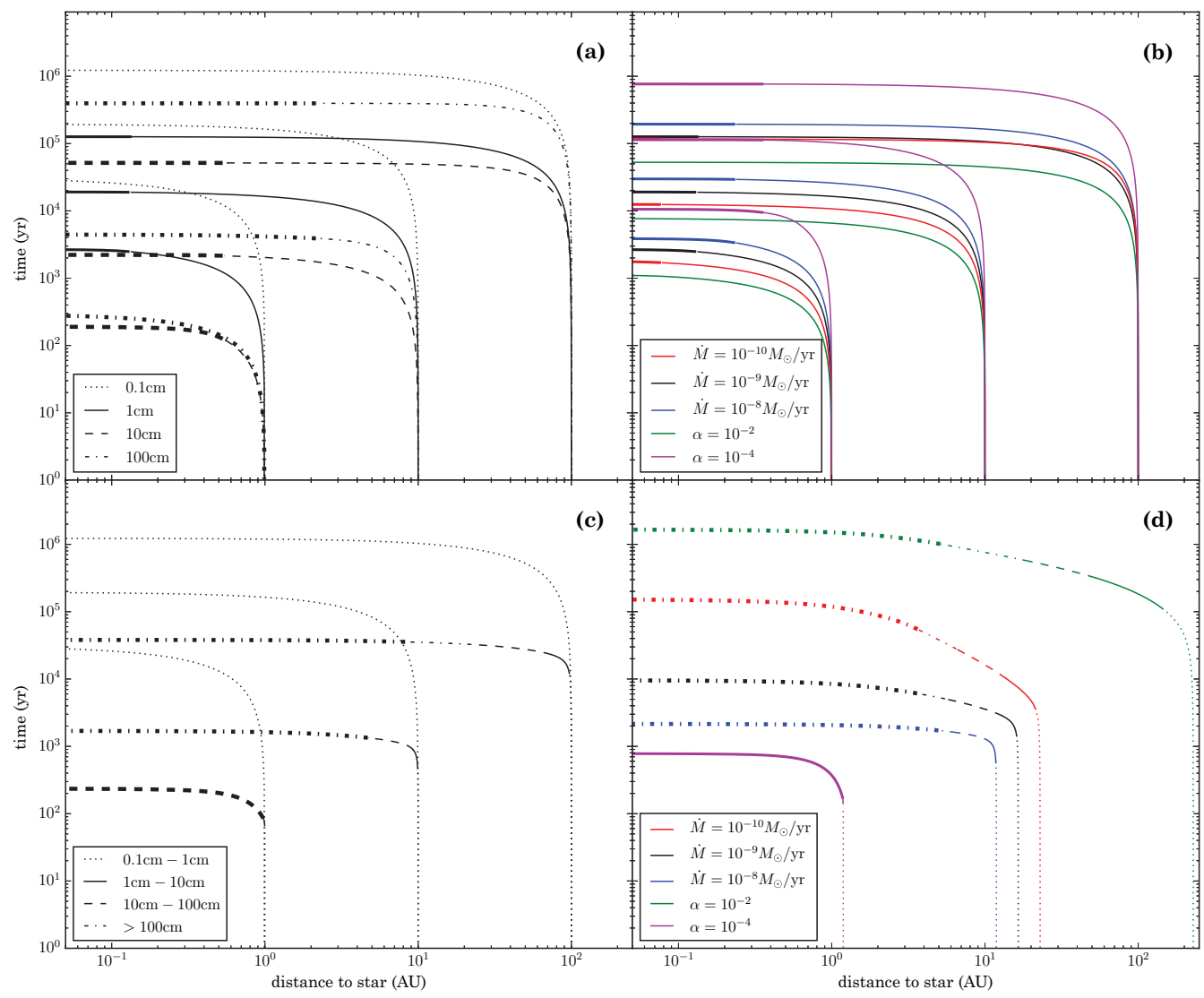

Figure 1. a: Radial drift timescale of pebbles with different fixed radial sizes starting from various disk radii in the fiducial disk model $\left(\dot{m}=10^{-9} M_{\odot} \mathrm{yr}^{-1}, \alpha=10^{-3}\right)$. Thicker lines show the Stokes drag regimes. b: Radial drift time scale of $1 \mathrm{~cm}$ fixed-sized pebbles in disk models with different accretion rates and $\alpha$ viscosities, varied around the fiducial model. c: Comparisons between fixed-size $0.1 \mathrm{~cm}$ pebbles and the growth model with same initial size. The different line styles indicate growth in pebble radius. d: Growth model drift times for initially $0.1 \mathrm{~cm}$ pebbles starting from the outer radius of the feeding zone $r_{1}$ for gap-opening mass planets (see text).

for a $0.1 \mathrm{~cm}$ pebble of constant size from this distance is $2.88 \times 10^{5} \mathrm{yr}$. The same pebble growing by sweeping up dust drifts in after $1.15 \times 10^{4}$ yr with final size of $233 \mathrm{~cm}$. This result is not very sensitive to the choice of inititial pebble size: a growing pebble starting with $0.01 \mathrm{~cm}$ radius has a drift time of $1.33 \times 10^{4} \mathrm{yr}$ and a very similar final size. Note, if $\epsilon_{p}$ is much smaller than our fiducial value, e.g., due to interception of pebbles by a population of outer disk planetesimals (Guillot et al. 2014), then this would increase the radius of the required feeding zone and thus lengthen the drift timescales.

These drift times thus set lower limits for the timescale of first, innermost planet formation in the IOPF model. Figure $1 \mathrm{~d}$ shows these times for various $\dot{m} \& \alpha$. They are shorter for disks that are denser, i.e., due to higher $\dot{m}$ or lower $\alpha$, given $M_{G}(\dot{m}, \alpha)$ and that the feeding zone is then smaller and pebble growth more efficient. These formation times may also be lower limits if pebble formation by dust grain coagulation (e.g., Birnstiel et al. 2012) is the rate limiting step, to be investigated in future work. Still, Fig. 1d shows IOPF requires $\alpha \lesssim 10^{-3}$, i.e., dead zone conditions, given observed disk lifetimes $\sim 1$ Myr. Variation in dead zone properties, e.g., from different disk midplane ionization 
rates by cosmic rays or radionuclide decay, could thus lead to a variety of planetary system formation mechanisms, perhaps helping to explain STIP vs. Solar System formation.

\section{References}

Armitage, P. J. 2010, Astrophysics of Planet Formation, Cambridge University Press, 2010 Birnstiel, T., Klahr, H., \& Ercolano, B. 2012, A\&A, 539, 148

Chatterjee, S. \& Tan, J. C. 2014, ApJ, 780, 53

Chiang, E. \& Laughlin, G. 2013, MNRAS, 431, 3444

Guillot, T, Ida, S. \& Ormel, C. W. 2014, arXiv: 1409.7328

Hands, T. O., Alexander, R. D., \& Dehnen, W. 2014, arXiv: 1409.0532

Hansen, B. M. S. \& Murray, N. 2012, ApJ, 751, 158

Hansen, B. M. S. \& Murray, N. 2013, ApJ, 775, 53

Zhu, Z., Stone, J. M., \& Rafikov, R. R. 2013 ApJ, 768, 143 